\title{
Etudes et simulations d'architectures de tiroirs à paramètres $S$ d'analyseurs de réseaux
}

\author{
Jean-François Favennec ${ }^{(1)}$, Cédric Quendo ${ }^{(2)}$, Eric Rius ${ }^{(2)}$, Christian Person ${ }^{(3)}$, Michel Cauterman ${ }^{(1)}$, \\ Ronan Abalain ${ }^{(1)}$, David Surget ${ }^{(1)}$, Fabien Astruc ${ }^{(1)}$, Sylvain Potet ${ }^{(1)}$, Sébastien Jacq ${ }^{(1)}$, \\ Youness Kadara ${ }^{(1)}$
}

(1) Ecole Nationale d’Ingénieurs de Brest - Technopôle Brest Iroise - CS 73862 - 29238 BREST Cedex 3

(2) Département d'Electronique - UFR Sciences et Techniques - Université de Brest - 6 avenue Le Gorgeu- CS 93837 - 29238 BREST Cedex 3

(3) Ecole Nationale Supérieure des Télécommunications de Bretagne - Technopôle Brest Iroise - CS 83818 29238 Brest Cedex 3

favennec@enib.fr

\begin{abstract}
RESUME : La mesure de circuits en hyperfréquences est un problème délicat. Nous souhaitons y sensibiliser les étudiants par l'étude et la simulation d'un tiroir à paramètres S d'un analyseur de réseaux ; qui est un appareil de mesure incontournable en hyperfréquences. La démarche proposée consiste dans un premier temps à travailler sur un dispositif constitué de composants idéaux. Malheureusement, la réalité est toute autre et les composants présentent toujours des défauts. On remplace donc certains composants idéaux par des modèles plus réalistes. On constate alors la dégradation de la qualité des mesures, qui seraient effectuées par un tel dispositif. A ce stade, il est demandé aux étudiants d'en analyser précisément les raisons ; et de proposer une nouvelle architecture du tiroir à paramètres $\mathrm{S}$ permettant d'obtenir des mesures de bonne qualité. C'est dans le cadre de travaux dirigés que cette étude d'un tiroir à paramètres $S$ est d’abord effectuée. Puis au cours de mini-projets les étudiants sont amenés à valider les résultats des travaux dirigés par des simulations dans le domaine temporel. Enfin, ils effectuent réellement la mesure d'un filtre sur un analyseur de réseaux.
\end{abstract}

Mots clés : hyperfréquence, analyseur de réseaux, tiroir à paramètres S, simulation dans le domaine temporel, miniprojets

\section{INTRODUCTION}

La démarche présentée dans cette communication a été initiée à l'ENIB. Cependant elle n'aurait pu aboutir sans la participation de collègues membres du département d'électronique de l'UBO et du département microondes de l'ENSTBr. En effet, c'est au travers de nombreux échanges que l'ensemble des problèmes techniques a été résolu. L'utilisation de simulateurs peut s'avérer complexe et parfois déroutante aussi est-il très important de pouvoir bénéficier du retour d'expérience de collègues travaillant sur les mêmes outils. D'autre part, sur le plan pédagogique, il est essentiel de soumettre une démarche originale aux critiques des collègues enseignant dans le même domaine. A ce titre ce fut très intéressant et enrichissant.

Ces enseignements s'adressent à des étudiants en dernière année de cycle ingénieurs ou en master 2 dans le cadre de modules de spécialisation en hyperfréquence. Ils prennent la forme de TD suivis de mini-projets. Ils nécessitent environ une quinzaine d'heures.

L'analyseur de réseaux est un appareil de mesure indispensable en hyperfréquences. Il est constitué d'une source, d'un synthétiseur de fréquences, d'un tiroir à paramètres $S$ et d'un dispositif permettant la mesure des signaux, ainsi que leur mise en forme pour leur représentation sur un écran. Le Dispositif Sous Test
(DST) est connecté entre les deux ports de l'analyseur de réseaux.

Nous nous intéressons au tiroir à paramètres S. Dans un premier temps, nous proposons en travaux dirigés l'étude d'une architecture classique simplifiée d'un tiroir à paramètres S. Elle est constituée d'un isolateur et de trois coupleurs contradirectifs, qui permettent d'extraire en différents endroits de la chaîne une petite partie du signal afin de déterminer les paramètres $\mathrm{S}$ du DST. Ces coupleurs présentent dans un premier temps une isolation infinie. Dans ce cas, il est possible d'extraire les paramètres $\mathrm{S}$ du DST, à partir des signaux issus de la chaîne. Cependant en réalité, un coupleur ne peut pas avoir une isolation infinie. Dans ce cas, on constate une dégradation importante de la qualité des paramètres $\mathrm{S}$ obtenus avec cette architecture. Toujours dans le cadre des travaux dirigés, les étudiants doivent alors proposer une nouvelle architecture d'un tiroir à paramètres $\mathrm{S}$ permettant l'utilisation de coupleurs réels.

La deuxième partie de l'étude prend la forme de miniprojets. Chaque binôme d'étudiants doit simuler dans le domaine temporel les tiroirs à paramètres $\mathrm{S}$ étudiés pendant les travaux dirigés. A chaque fois il doit en extraire les paramètres $S$ du filtre utilisé comme DST. Ces mini-projets permettent non seulement de valider le travail effectué auparavant en TD, mais de plus ils sont une excellente initiation aux simulations de dispositifs dans le domaine temporel. Enfin, il est proposé aux 
étudiants une initiation à la mesure sur analyseur de réseaux, au cours de laquelle ils mesurent un filtre microruban.

\section{LE TIROIR A PARAMETRES S}

Le tiroir à paramètres S [1] aiguille une onde incidente sur un des ports de l'analyseur de réseaux, c'est-à-dire sur un des accès du DST. Cette énergie incidente est en partie réfléchie, transmise ou perdue par le DST.

Des commutateurs permettent de basculer l'onde incidente soit sur le port 1 ou soit sur le port 2 de l'analyseur. Dans le cas du port 1, on mesure les paramètres $S_{11}$ et $S_{21}$ du DST. Dans le cas du port 2, on mesure alors les paramètres $S_{22}$ et $S_{12}$ du DST. De la sorte, il est possible de mesurer les quatre paramètres $S$ du quadripôle sous test sans avoir à le démonter.

On représente sur la figure 1 le schéma de principe simplifié du tiroir à paramètres $\mathrm{S}$ de l'analyseur de réseaux, dans le cas où les commutateurs (non représentés) sont positionnés pour mesurer les paramètres $S_{11}$ et $S_{21}$ du DST.

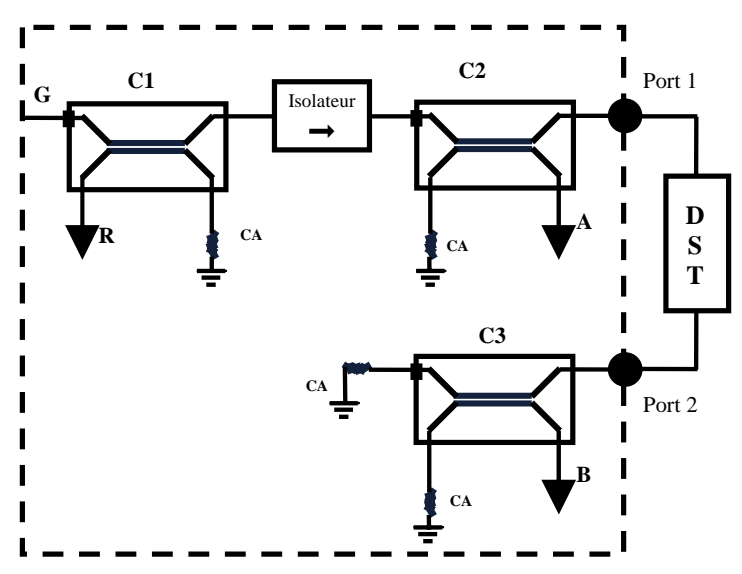

fig 1 : Schéma de principe simplifié du tiroir à paramètres $S$ mesure des paramètres $S_{11}$ et $S_{21}$ du DST.

Les énergies réfléchie et transmise par le DST retournent dans le tiroir à paramètres $S$ de l'analyseur de réseaux. Une partie des ondes réfléchie et transmise est alors prélevée grâce aux coupleurs contradirectifs (C2 et C3). La mesure des caractéristiques (puissance et phase) de ces ondes prélevées $\mathrm{A}$ et $\mathrm{B}$ permet de déterminer les paramètres S du DST.

A noter qu'il est nécessaire de connaître les caractéristiques de l'onde incidente. C'est pourquoi une faible proportion de l'onde incidente est aussi prélevée par le coupleur C1. Il s'agit de la sortie R. Un isolateur protège la source $\mathrm{G}$ des réflexions sur le DST, il permet aussi d'assurer sur $\mathrm{R}$ un signal provenant uniquement d'un prélèvement du signal d'entrée du tiroir.
Dans un premier temps nous considérons des coupleurs contradirectifs, idéaux [2], identiques, dont le schéma synoptique et la matrice $S$ sont donnés sur la figure 2 . Ainsi lorsque le coupleur est excité par l'accès 1, l'énergie se répartit sur l'accès 2 (voie directe) et sur l'accès 3 (voie couplée). Aucune puissance n'est transmise vers l'accès 4 (isolation et directivité infinies).

$$
\left[S_{C}\right]=\left[\begin{array}{cccc}
0 & S_{21}^{C} & S_{31}^{C} & 0 \\
S_{21}^{C} & 0 & 0 & S_{42}^{C} \\
S_{31}^{C} & 0 & 0 & S_{43}^{C} \\
0 & S_{42}^{C} & S_{43}^{C} & 0
\end{array}\right]
$$

fig 2 : Schéma synoptique et matrice $S$ du coupleur directionnel présentant une isolation infinie.

Les étudiants établissent donc en TD les expressions analytiques des ondes sortantes sur R, A et B en fonction de l'onde entrante $\mathrm{a}_{\mathrm{G}}$ dans le tiroir à paramètres $\mathrm{S}$.

$$
\begin{aligned}
& b_{R}=S_{31}^{C} \cdot a_{G} \\
& b_{A}=\left(S_{21}^{C}\right)^{2} S_{11}^{F} S_{42}^{C} \cdot a_{G} \\
& b_{B}=\left(S_{21}^{C}\right)^{2} S_{21}^{F} S_{42}^{C} \cdot a_{G}
\end{aligned}
$$

$S_{11}^{F}$ et $S_{21}^{F}$ sont respectivement les coefficients de réflexion et de transmission du DST, qui ici est un filtre. La connaissance des ondes $b_{A}, b_{B}$ et $b_{R}$, ainsi que des paramètres $\mathrm{S}$ des coupleurs, permet donc de déterminer $S_{11}^{F}$ et $S_{21}^{F}$.

En réalité nos coupleurs ne présentent pas une isolation infinie [2]. Une petite partie de l'onde incidente est aiguillée sur la voix isolée, ainsi $S_{41}^{C}$ et $S_{32}^{C}$ ne sont plus nuls. $S_{41}^{C}$ et $S_{32}^{C}$ présentent cependant une amplitude très faible. Les coupleurs sont toujours identiques, réciproques et adaptés à tous leurs accès.

La matrice S du coupleur prend donc la forme suivante :

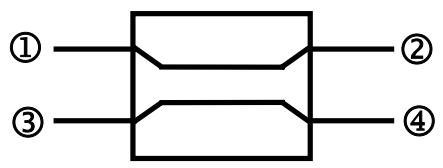

$$
\left[S_{C}\right]=\left[\begin{array}{cccc}
0 & S_{21}^{C} & S_{31}^{C} & S_{41}^{C} \\
S_{21}^{C} & 0 & S_{32}^{C} & S_{42}^{C} \\
S_{31}^{C} & S_{32}^{C} & 0 & S_{43}^{C} \\
S_{41}^{C} & S_{42}^{C} & S_{43}^{C} & 0
\end{array}\right]
$$

fig 3 : Schéma synoptique et matrice $S$ du coupleur directionnel ne présentant pas une isolation infinie. 
Un calcul des ondes obtenues en sortie des coupleurs permet de constater que seule la sortie A est "polluée" par un signal supplémentaire ne provenant pas de la réflexion sur le DST, à laquelle il vient se superposer :

$$
b_{A}=\left(\left(S_{21}^{C}\right)^{2} S_{11}^{F} S_{42}^{C}+S_{21}^{C} \cdot S_{41}^{C}\right) a_{G}
$$

En effet il existe ici une énergie parasite issue du défaut d'isolation des coupleurs. Bien que cette énergie soit faible, elle peut être importante par rapport à l'énergie issue de la réflexion sur le filtre notamment dans la bande passante. En effet dans la bande passante, le filtre est adapté d'où une réflexion de faible ampleur sur ses accès. De plus seule une petite portion de cette réflexion sera aiguillée vers la sortie A par le coupleur C2.

Ce défaut d'isolation présent sur tous les coupleurs réels dégrade donc la mesure des coefficients de réflexion des DST.

Toujours en travaux dirigés, les étudiants doivent imaginer une architecture alternative permettant de pallier ce problème. Plusieurs solutions sont possibles. Nous présentons l'une d'entre elles sur la figure 4 :

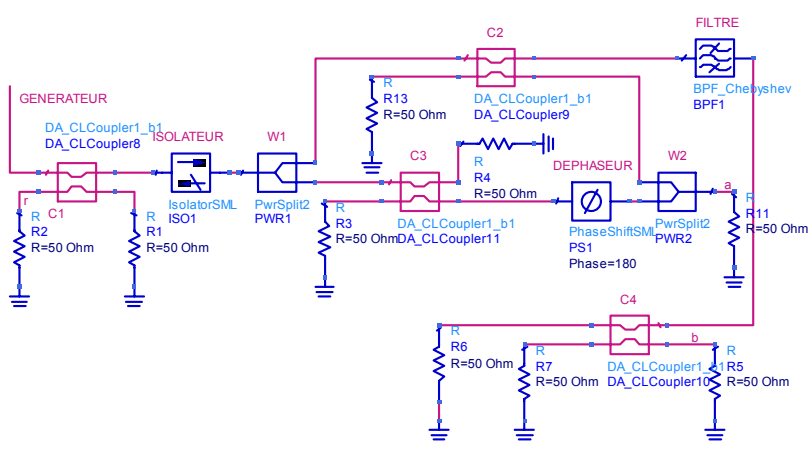

fig 4 : Proposition d'architecture alternative

Elle est constituée de quatre coupleurs de deux diviseurs de puissance de Wilkinson, d'un isolateur, ainsi que d'un déphaseur de $180^{\circ}$. Le principe est de prélever sur la sortie isolée du coupleur C3 un signal identique, en amplitude et en phase, au signal perturbateur qui viendra se superposer au signal issu de la réflexion sur le filtre. Il suffit ensuite de déphaser de $180^{\circ}$ ce signal, et de l'injecter dans le diviseur de Wilkinson W2. Sur l'autre branche de W2 on injecte le signal provenant du défaut d'isolation sur C2 et le signal issu de la réflexion sur le filtre. Aux entrées de W2, les deux signaux nés des défauts d'isolation des coupleurs C2 et C3 sont en opposition de phase. Ils sont donc recombinés de façon destructive dans le diviseur W2. Sur la sortie A de W2, il ne reste donc que le signal provenant de la réflexion sur le filtre.

Bien sûr cette architecture est relativement complexe et sans doute très difficile à réaliser, compte tenu entre autres des défauts d'adaptation de l'ensemble des composants. Cependant, elle a permis aux étudiants qui l'ont proposée d'associer un nombre conséquent de fonctions élémentaires très utilisées en hyperfréquence et d'imaginer une solution à un problème très gênant. D’autres architectures alternatives ont été proposées, certaines utilisent d'autres composants comme des anneaux hybrides.

Pendant les séances de travaux dirigés, nous retenons une proposition d'architecture alternative pour laquelle les étudiants déterminent notamment les ondes sortantes aux accès $A, B$ et $R$; mettant ainsi en évidence la pertinence de cette nouvelle architecture.

\section{DETERMINATION DES PARAMETRES S DU FILTRE PAR DES SIMULATIONS TEMPORELLES}

Après avoir étudié en travaux dirigés le principe d'un tiroir à paramètres $S$, nous proposons aux étudiants de simuler le dispositif de la figure 1 . On considère d'abord des coupleurs sans défaut d'isolation, puis on les remplace par d'autres coupleurs présentant ce défaut. Ces simulations sont effectuées dans le cadre de miniprojets. Le simulateur utilisé est le logiciel ADS Agilent $^{\mathrm{TM}}$. L'objectif de la démarche est de déterminer les modules des paramètres $S_{11}^{F}$ et $S_{21}^{F}$ du filtre sur une plage de fréquence par un ensemble de simulations temporelles. Pour ce faire on a accès aux tensions en A, $B$ et $R$. Le circuit est alimenté par un générateur délivrant un signal sinusoïdal de $1 \mathrm{~mW}$ de puissance.

Dans un premier temps, les étudiants doivent déterminer comment extraire des paramètres $\mathrm{S}$ à partir de tensions sinusoïdales.

Pour un quadripôle, le module au carré du coefficient de transmission entre la sortie 2 et l'entrée 1 est lié aux puissances sortante à l'accès 2 et incidente à l'accès 1 par :

$$
\left|S_{21}\right|^{2}=\frac{P_{\text {sor tante } 2}}{P_{\text {incidente } 1}}
$$

Un rapide calcul permet de déterminer $\left|S_{21}\right|$ en fonction des tensions crêtes accessibles aux accès 1 et 2 du

quadripôle : $\left|S_{21}\right|=\frac{U_{\text {crête 2 }}}{U_{\text {crête } 1}}$

Il est donc nécessaire d’accéder aux tensions crêtes des signaux.

Les étudiants dessinent, sous ADS, un premier tiroir à paramètres $S$ (figure 5) basé sur des coupleurs présentant une isolation et une directivité infinies. Les coupleurs ont un facteur de couplage $\mathrm{C}=16 \mathrm{~dB}$. Une faible partie de la puissance est donc prélevée par chaque coupleur. Ils sont "réalisés" par des lignes couplées "quart d’onde" idéales. La première étape consiste donc à déterminer les impédances caractéristiques en mode pair et impair des lignes couplées pour obtenir ce facteur de couplage. Ceci est très facilement réalisable en utilisant l'outil LineCalc d'ADS, ou en se référant à la littérature [2]. 
Le filtre doit être "mesuré" sur une plage de fréquence. A chaque point de fréquence, on fait une simulation dans le domaine temporel avec l'outil "Transient". Le balayage en fréquence est effectué par le composant "Parameter Sweep", qui permet de modifier la fréquence du générateur. Afin de remonter aux paramètres $S$ du filtre par une lecture des tensions crêtes en A, B et R ; il est nécessaire de caractériser les coupleurs pour chaque point de fréquence. C’est pourquoi, simultanément on simule à part un coupleur.

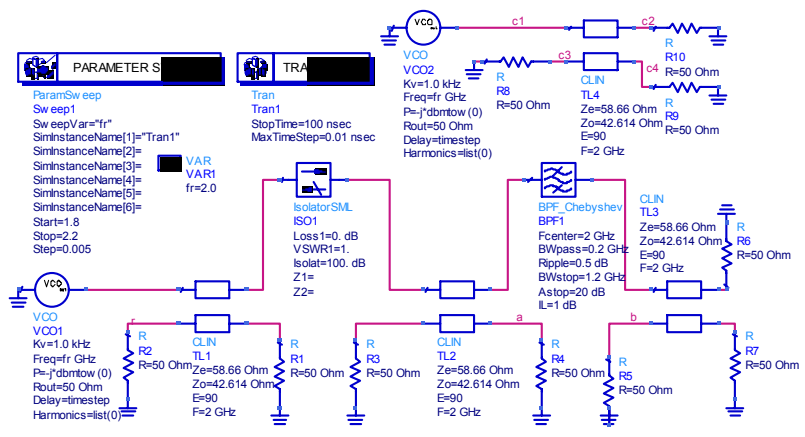

fig 5 : Tiroir à paramètres $S$ sous ADS - Coupleurs à isolation infinie

Une fois l'ensemble des simulations temporelles effectuées, il faut en extraire les modules de $S_{11}^{F}$ et $S_{21}^{F}$. Pour ce faire, les étudiants doivent déterminer, puis implanter dans la fenêtre d'ADS permettant de visualiser les résultas un ensemble de formules. D’abord celles qui permettent d'obtenir, pour chaque simulation temporelle, les tensions crêtes des signaux du tiroir à paramètres $\mathrm{S}$ et du coupleur,
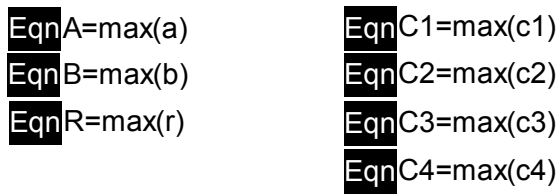

puis un autre jeu d'expressions qui donnent accès aux paramètres $\mathrm{S}$ du filtre :

Eqn $A R=20^{*} \log (\mathrm{A} / \mathrm{R}) \quad$ EqnFIITRE_S21 $=\mathrm{BR}-2^{*} \mathrm{~S} 21 \mathrm{C}$
Eqn $\mathrm{BR}=20^{*} \log (\mathrm{B} / \mathrm{R}) \quad$ EqnFIITRE_S11 $=\mathrm{AR}-2^{*} \mathrm{~S} 21 \mathrm{C}$
EqnS21C $=20^{*} \log (\mathrm{C} 2 / \mathrm{C} 1)$
EqnS31C $=20^{*} \log (\mathrm{C} 3 / \mathrm{C} 1)$
EqnS41C $=20^{*} \log (\mathrm{C} 4 / \mathrm{C} 1)$

Nous choisissons de travailler sur un filtre passe bande de Tchebycheff d'ordre 5, présentant une ondulation de $0,5 \mathrm{~dB}$ et des pertes d'insertion de $1 \mathrm{~dB}$. Ce filtre a une fréquence centrale de $2 \mathrm{GHz}$ et une bande passante de $200 \mathrm{MHz}$. Le filtre est simulé entre 1,8 GHz et 2,2 GHz.

Le filtre est d'abord simulé seul sous ADS avec l'outil de simulation fréquentiel "S Parameters" (figure 6).

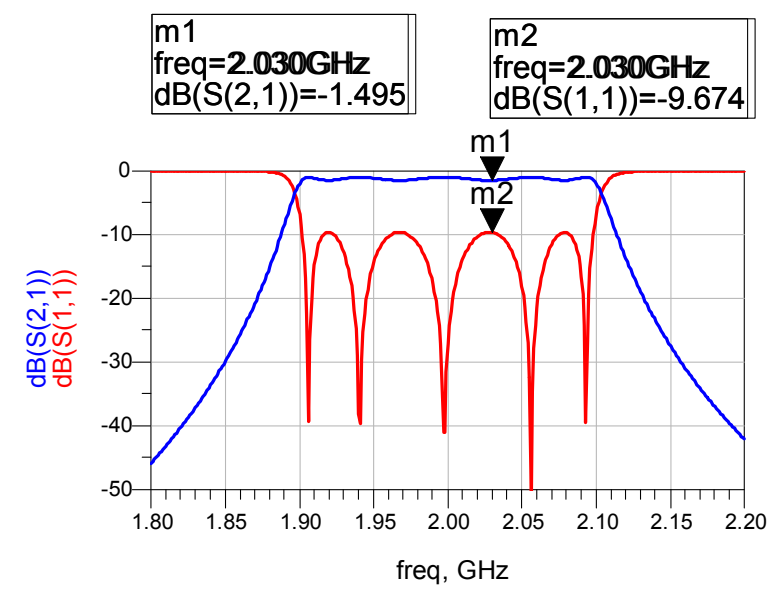

fig 6 : Réponses en amplitude de la transmission $\left(S_{21}\right)$ et de la réflexion $\left(S_{11}\right)$ du filtre obtenues par simulation fréquentielle

Ces deux réponses obtenues par un outil de simulation fréquentiel, prévu pour obtenir des paramètres $S$, servent pour la suite de références pour juger de la qualité des simulations temporelles et des architectures testées.

Le tiroir à paramètres $\mathrm{S}$ de la figure 5 est ensuite simulé dans le domaine temporel. Nous rappelons qu'il inclut des coupleurs présentant une isolation infinie. Les paramètres $\mathrm{S}$ du filtre sont extraits des simulations temporelles et présentés sur la figure 7 :

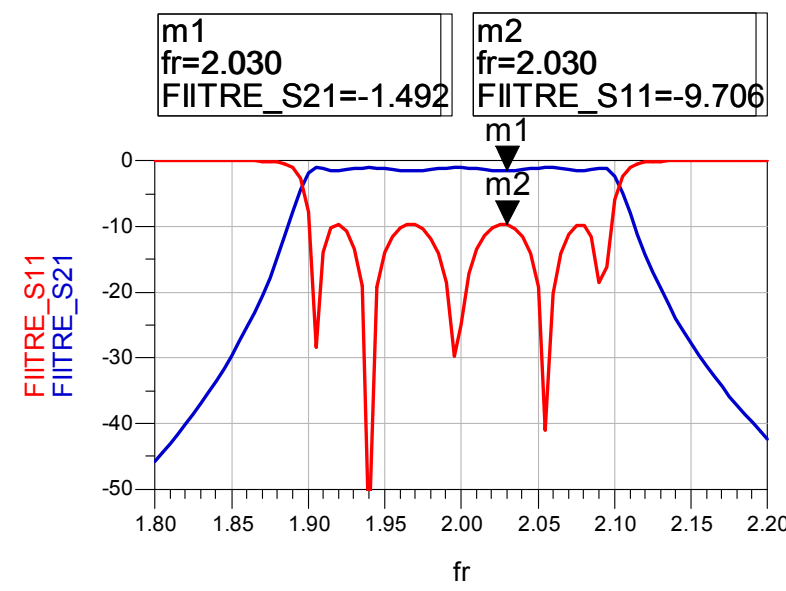

fig $7:\left|S_{21}\right|_{d B}$ et $\left|S_{11}\right|_{d B}$ du filtre extraites des simulations temporelles du tiroir à paramètres $S$ incluant des coupleurs à isolation infinie

Nous constatons en les comparant à ceux de la figure 6 que les résultats obtenus (figure 7 ) sont de très bonne qualité. Ainsi le tiroir à paramètres $\mathrm{S}$ de la figure 5 (coupleurs à isolation infinie) simulé dans le domaine temporel permet d'extraire sans problème les paramètres $\mathrm{S}$ du filtre. Cette simulation permet de vérifier aussi que la procédure de simulation fonctionne correctement. Les étudiants remplacent alors les coupleurs du circuit de la figure 5 par des modèles de coupleurs plus réalistes présentant donc une isolation finie. Les coupleurs ont les caractéristiques suivantes à $2 \mathrm{GHz}$ : 
- $\quad$ Facteur de couplage $\mathrm{C}=16 \mathrm{~dB}$

- Directivité : $\mathrm{D}=6,53 \mathrm{~dB}$

- Isolation : $\mathrm{I}=22,53 \mathrm{~dB}$

- $\quad$ Pertes d'insertion IL = 0,183 dB

- TOS $=1,04$

Ils sont "réalisés" par des lignes microruban couplées "quart d’onde". Le substrat utilisé a une permittivité effective $\varepsilon_{\mathrm{r}}=3,5$ et une épaisseur de $762 \mu \mathrm{m}$. Nous tenons aussi compte des pertes diélectriques $\operatorname{tg}(\delta)=0,003$; et nous considérons une conductivité des lignes de $41.10^{6} \mathrm{~S} / \mathrm{m}$.

La simulation temporelle est lancée et les résultats sont présentés sur la figure 8 :
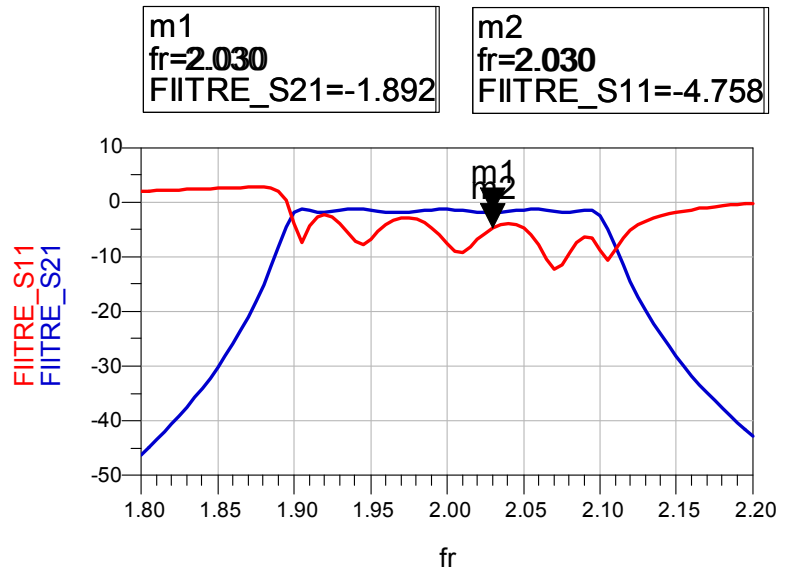

fig 8: $\left|S_{21}\right|_{d B}$ et $\left|S_{11}\right|_{d B}$ du filtre extraites des simulations temporelles du tiroir à paramètres $S$ incluant des coupleurs à isolation finie

On constate immédiatement que la réflexion est de très mauvaise qualité. Comme prévu, le signal arrivant sur la sortie A est pollué par le signal issu du défaut d'isolation de C2. Nous avons conservé ici un traitement des données identique à celui utilisé dans le cas de coupleurs présentant une isolation et une directivité infinies. On constate que ce n'est pas satisfaisant, notamment dans la bande passante et en dessous, où nous avons une réflexion supérieure à $0 \mathrm{~dB}$; ce qui physiquement est impossible. Il faut donc tenir compte de ce défaut d'isolation soit par un traitement numérique adéquat soit en modifiant l'architecture. Par contre la transmission est très proche de celle obtenue avec la simulation fréquentielle. La différence de niveau des pertes provient des pertes dans les coupleurs.

En ce qui concerne la phase des paramètres $S$, il n'existe pas dans ADS d'outil permettant de déterminer en simulation temporelle l'écart de phase entre deux signaux. Nous avons donc créé ce composant. Il est basé sur le "time delta", qui détermine l’écart de temps entre deux signaux. Un paramétrage judicieux (figure 9) permet d'obtenir sur la sortie 3, chargée par 1 Ohm, une tension pour laquelle chaque $\mu \mathrm{V}$ correspond a un déphasage de $1^{\circ}$ des signaux d'entrée.

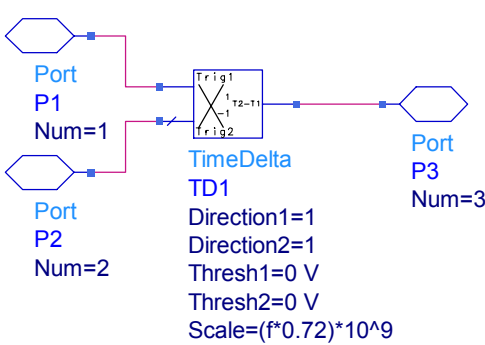

fig 9 :Comparateur de phase

Nous avons testé avec succès cet outil sur différents composants (déphaseur, gyrateur ...). Il sera prochainement utilisé sur des filtres par des étudiants en mini-projet.

Il n'est pas envisageable au cours des séances de miniprojets de procéder aux simulations des architectures alternatives. En effet, lorsque les dispositifs à simuler sont assez conséquents, les simulations temporelles peuvent être assez difficiles à maîtriser au niveau des réglages de l'outil "Transient". De plus dans ce cas, les durées de simulation sont assez importantes.

Pour compléter cet enseignement axé sur les analyseurs de réseaux, la mesure d'un filtre est proposée aux étudiants. Cette initiation permet notamment de découvrir les procédures de calibration nécessaires à l'utilisation de ces appareils de mesure particuliers.

\section{CONCLUSION}

La démarche que nous venons de présenter, a pour ambition de permettre aux étudiants en hyperfréquence d'aborder non seulement la problématique de la mesure et d'appréhender toute la difficulté d'obtenir des résultats fiables; mais aussi d'acquérir une expérience en simulation dans le domaine temporel. Enfin, ils peuvent manipuler sur un analyseur de réseaux, qui est un appareil de mesure incontournable en hyperfréquence. Cet enseignement conçu sous la forme de travaux dirigés et de mini-projets permet de mettre en œuvre les acquis du cours d'hyperfréquence, sur la problématique de la mesure qui est un sujet essentiel dans les métiers d'ingénieur ou de chercheur.

Notre prochain objectif est la prise en compte de la calibration [3], qui est une procédure systématique permettant d'éliminer les contributions des câbles et des connecteurs.

\section{Bibliographie}

[1] "Operating and Service Manual" AgilentTechnologies, 85047A S-Parameter Test Set, Manufacturing Part Number: 85047-90001, juillet 1996,

[2] David M. Pozar. "Microwave Engineering". Wiley, second edition, p. 354 - 357.; 383 - 394

[3] K.C. Gupta, R. Garg, R. Chadla "Computer-aided design of microwave circuits" Artech House, p : 295-324 